Sociologie et sociétés

\title{
Impact des publics raccrocheurs sur la culture organisationnelle des services de l'éducation des adultes : une étude de cas
}

\section{The Impact of Groups Reintegrating Society on the Organizational Culture of Adult Education Services: A Case Study}

\section{Louis TOUPIN}

Volume 20, numéro 1, printemps 1988

La sociologie hors université

URI : https://id.erudit.org/iderudit/001797ar

DOI : https://doi.org/10.7202/001797ar

Aller au sommaire du numéro

Éditeur(s)

Les Presses de l'Université de Montréal

ISSN

0038-030X (imprimé)

1492-1375 (numérique)

Découvrir la revue

Citer cet article

TOUPIN, L. (1988). Impact des publics raccrocheurs sur la culture organisationnelle des services de l'éducation des adultes : une étude de cas.

Sociologie et sociétés, 20(1), 61-70. https://doi.org/10.7202/001797ar

\section{Résumé de l'article}

Après trois ans d'existence, les Services de l'éducation des adultes de la Commission scolaire régionale Blainville-Deux-Montagnes examinent l'impact que le programme de formation pour jeune bénéficiaire de l'aide sociale a eu sur l'organisation scolaire. On a réalisé une étude de cas, qualitative et exploratoire. Elle permet d'interpréter et d'appréhender de façon contextuelle un certain nombre de problèmes actuels tel celui du rapport entre jeunes et adultes et le recours de plus en plus fréquent aux programmes centrés sur une clientèle dans l'organisation scolaire. 


\section{Impact des publics raccrocheurs sur la culture organisationnelle des services de l'éducation des adultes: une étude de} cas

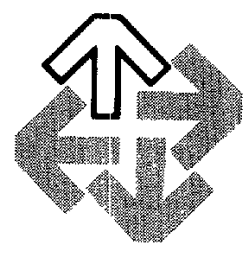

\section{LOUIS TOUPIN*}

\section{CONTEXTE DE LA RECHERCHE}

On a cru trop vite que le discours néo-conservateur des années 80 allait remettre en question l'État-providence. En fait, en brandissant l'épouvantail d'une remise en cause des mesures de protections sociales, le discours rendait possible l'existence d'une pilule moindre, plus facile à avaler, mais toute aussi amère. Ainsi, plutôt que d'un État-providence, il faudrait peut-être maintenant parler d'un «État-référence». La plupart des programmes sociaux ont été maintenus à ceci près qu'ils définissent davantage les publics, selon le jargon les «clientèles cibles», auxquels ils s'adressent. Dorénavant, pour avoir accès à telle ou telle mesure sociale, il faut rencontrer des critères d'admissibilité de plus en plus précis, différenciés et priorisés. Cette critériologie est le résultat direct de la crise fiscale que traversent la plupart des sociétés industrialisées. Cette crise impose des contraintes lourdes sur les dépenses de fonds publics. C'est dans ce contexte que la plupart des gouvernements se sont livrés à de multiples évaluations de programmes afin de les rationaliser davantage, d'éviter les dédoublements et d'en vérifier l'efficacité.

C'est dorénavant au nom de ce dernier critère, l'efficacité, que les gouvernements exigent des programmes capables d'atteindre vraiment ceux et celles auxquels ils sont destinés. Avec des programmes ainsi définis, la recherche organisationnelle a fort à faire pour identifier, catégoriser, inventorier, typologiser, bref «traquer» et «disséquer» ces inasignables destinataires. Il faut connaître leur mode de vie, leur réseau d'appartenance, leurs valeurs, leurs caractéristiques, leurs nombres, leurs localisations et ainsi de suite. En filigrane, on sent une inquiétude poindre de ces études: une société duale (Servoin et Duchemin, 1986), composée pour une part d'individus «répertoriables» productifs et compétitifs, et pour l'autre d'individus «non répertoriables» exclus et marginaux, est-elle en train de naître sous nos yeux?

* Louis Toupin est agent de recherche dans le domaine de l'éducation, notamment l'éducation des adultes, depuis 10 ans. Au cours de ces années, ses travaux ont principalement porté sur les politiques en éducation, le développement professionnel des enseignants et enseignantes, la pédagogie en milieu carcéral et l'éducatique. De plus, il est actuellement inscrit au programme de doctorat en sciences de l'éducation à l'université de Montréal. Sa thèse portera sur le domaine des transferts de connaissances. 
Parmi les programmes clientèles mis en place aux cours des dernières années, plusieurs ont donné la priorité aux jeunes. C'est au cours des années 60 que l'on a commencé à éclairer le conflit jeunes-adultes que traversent les sociétés occidentales. À ce moment-là, on parlait de conflits de générations (generation gap). À cette vision quelque peu simpliste du problème, l'évolution du contexte social ainsi que diverses études ont permis d'attirer l'attention des chercheurs, des gouvernements et du public sur certains problèmes spécifiques à la jeunesse. Parmi ces problèmes, les conséquences psychologiques et sociales du chômage chez les jeunes (Demers, 1983) et l'insertion sociale et professionnelle des jeunes (Grégoire, 1981) ont reçu un éclairage prioritaire, parfois dramatique (Hébert et Yuzyk, 1986).

En 1983, faisant suite à la crise économique amorcée en 1982, le gouvernement du Québec rendait public un ensemble de mesures dont l'objectif général était la relance économique. Une priorité se dégage alors de ces mesures: les jeunes. Dans un marché du travail en bonne partie institutionnalisé et protégé, et où les emplois sont en chute libre, il fallait à tout prix minimiser l'impact de la crise économique sur ceux-ci. Cependant, les préoccupations néoconservatrices n'étaient pas absentes de ce plan de relance. En effet, parmi les mesures annoncées, l'une d'elle s'adressait aux jeunes âgés de 18 à 30 ans bénéficiaires de l'aide sociale. Entre autres, il s'agissait d'exercer une pression financière sur ces jeunes pour qu'ils s'inscrivent à l'un ou l'autre des programmes visant à relever leur employabilité, dont la formation scolaire.

Ce n'est qu'un an plus tard, en 1984, que la nature fiscale de cette mesure est clairement apparue. Dans le livre blanc sur la fiscalité des particuliers, le gouvernement du Québec annonce une toute nouvelle stratégie de lutte contre la pauvreté:

L'approche actuelle qui consiste à verser de façon automatique des prestations à tous ceux qui se retrouvent au-dessous du seuil de la pauvreté définie par l'État devient de moins en moins efficace et de plus en plus coûteuse. (p. 202)

Dorénavant, propose le livre blanc, les plus démunis devront entreprendre des démarches pour améliorer eux-mêmes leur sort plutôt que de s'en remettre à des moyens qui accroissent leur dépendance à l'égard de l'État. Ce à quoi le gouvernement songe explicitement, c'est à une réforme en profondeur de l'aide sociale. Entretemps, les mesures prises envers les jeunes bénéficiaires de l'aide sociale constituent, selon l'expression employée dans le livre blanc, un laboratoire privilégié pour cette éventuelle réforme de l'aide sociale qui n'a toujours pas vu le jour.

\section{POSITION DU PROBLÈME}

L'éducation des adultes des années 80 , contrairement à la décennie précédente, voit la très large majorité de ses publics usagers provenir de multiples programmes clientèles: pour accidentés du travail, pour personnes handicapées, pour femmes désirant effectuer un retour sur le marché du travail, pour la formation en entreprise et ainsi de suite. Parmi ces programmes, celui s'adressant aux jeunes bénéficiaires de l'aide sociale a été, et de loin, celui qui a laissé une empreinte profonde sur les Services de l'éducation des adultes (S.E.A.) des Commissions scolaires du Québec.

Un survol de la littérature récente se rapportant aux publics raccrocheurs (i.e. dropout) révèle que la plupart des études s'intéressent soit aux caractéristiques psychosociales de ces jeunes (Cungemi et Coan, 1973; Dubois, 1985; Blackburn, 1986; Forrest, 1986), ou bien aux causes de leurs abandons scolaires (Cungemi et Coan 1973; Hedman et Horning, 1983), ou encore à l'efficacité des méthodes d'intervention (Batimon, 1985; Campeau, 1985; Lemonde, 1986; Blackburn, 1986; Justiz et Kameen, 1987; Jones et Marockie, 1987).

Il n'existe pas d'études connues, organisationnelles ou scientifiques, qui font état de l'adaptation des institutions d'enseignement à cette «nouvelle clientèle». Pourtant, et c'est là un thème valorisé par l'approche systémique, il existe une interaction à double sens entre un organisme et son environnement. Un organisme qui se voit confier de «nouvelles clientèles» cherchera à mieux les connaître. En même temps, il est tout aussi pertinent de s'interroger, lorsque l'impact des «nouvelles clientèles» est important, sur les modifications et changements 
que l'organisme a dû (ou devrait) faire pour s'adapter à celles-ci. Une telle étude peut être révélatrice de la dynamique sous-jacente, c'est-à-dire intentionnelle, qui préside à la mise en place de mesures politiques souvent présentées comme généreuses et responsables.

C'est ce problème que les S.E.A. de la Cơmmission scolaire régionale Blainville-DeuxMontagnes ont retenu pour fin d'analyse en 1986-1987. Il s'agissait alors d'évaluer l'impact des publics raccrocheurs sur l'organisaion scolaire pour en tirer des conclusions pratiques et théoriques.

\section{MÉTHODOLOGIE}

Le problème de la recherche organisationnelle est souvent posé en ces termes: est-ce que la recherche organisationnelle peut où devrait être scientifique? C'est là un parti-pris de départ qui, à terme, ne peut rien donner de positif. Plutôt, il semble préférable de poser le problème de la façon suivante: est-ce que les diverses approches scientifiques connues (i.e. positiviste, systémique, historique et interprétative) peuvent être adaptées aux exigences particulières de la recherche organisationnelle? Il s'agit alors de trouver la meilleure théorie et la meilleure méthode qui conviennent à l'étude du problème de recherche (Schmidt, 1987).

L'impact des publics raccrocheurs sur des organismes d'enseignement n'ayant pas fait l'objet d'étude particulière, il devenait évident qu'une étude de ce genre ne pouvait être que qualitive et exploratoire. De même, ce qui préoccupait alors les S.E.A. était de donner un sens aux interactions entre ses membres et les publics raccrocheurs. Ces interactions prenant la forme de nouvelles règles, normes et pratiques langagières, l'approche interprétative apparaissait comme la plus adéquate pour rendre compte de l'objet d'étude.

L'approche interprétative a reçu des travaux de Michael Polanyi (1958) d'importantes contributions théoriques. Entre autres, Polanyi défend la thèse que la science repose sur les connaissances personnelles des scientifiques et leur habileté à produire de nouveau cadre interprétatif permettant de solutionner, de façon imaginative, les problèmes pratiques et langagiers que pose la recherche scientifique. La production de connaissance scientifique apparaît donc, chez Polanyi, comme le résultat de l'imagination conceptuelle, de l'évaluation et de l'appréciation des scientifiques. Plusieurs hésiteraient à appliquer le même raisonnement à des connaissances issues de la pratique. L'idée étant que ce sont les scientifiques qui produisent les connaissances et les praticiens qui les appliquent. Ce point de vue restrictif peut être remis en question. Les praticiens, ici des gestionnaires, formateurs ou formatrices et professionnels de l'enseignement, n'appliquent pas de recettes de cuisine ou des connaissances toutes faites. Ils font preuve d'un esprit tout aussi inventif dans leur façon d'utiliser des connaissances «produites» en les adaptant aux problèmes pratiques et théoriques que pose l'intervention dans un contexte. Il y a là production d'un autre type de connaissance (De Martini et Whitbeck, 1986). Il s'agit d'une connaissance, connaissance «clinique» diront certains, résultant de l'intégration de concepts théoriques, à des dimensions stratégiques, symboliques et intersubjectives inhérentes aux pratiques sociales.

Le chercheur rattaché à une organisation $a$, dans ce contexte, la délicate mission de traduire sous forme de discours le sens qui est présent dans les pratiques. Cependant, le praticien est, face à l'objet d'étude, situé dans une première distance alors que le chercheur l'est dans une seconde distance. Celui-ci n'est pas, à proprement parler, le «fidèle» porteparole du sens que les acteurs donnent à leur pratique. Le chercheur est plutôt préoccupé de construire les pratiques comme objet d'étude en visant leur intelligibilité. Par sa propre pratique de recherche, il peut aussi ajouter légitimement un sens à celui qui est déjà là chez les praticiens.

L'approche interprétative a ainsi fourni le cadre théorique permettant de reconstruire le sens des interactions entre l'organisation et les jeunes raccrocheurs. Pour opérationnaliser une telle approche mettant l'accent sur l'explication phénoménologique, archétypale ou symbolique des acteurs, l'analyse de cas s'est avérée la plus pertinente. Selon Robert K. Yin (1981, 1984), l'analyse de cas est particulièrement indiquée lorsqu'il s'agit d'examiner un phénomène nouveau dans son contexte, notamment lorsque les frontières entre phénomène et contexte ne 
sont pas évidentes et parfois inséparables. Les chercheurs utilisant l'analyse de cas doivent prêter attention aux cinq composantes suivantes: la définition du problème, le design de la recherche, la collecte de données, l'analyse et l'interprétation des données ainsi que la présentation du rapport (Yin et al., 1985). Ces composantes varieront selon que le but de l'étude de cas est de produire des connaissances pratiques ou théoriques (ou les deux). Ce sont ces cinq composantes qui vont être rapidement examinées.

Le problème de recherche en étant un d'impact de nouvelles clientèles sur une organisation spécifique, le design de la recherche fut établi en conséquence. Ainsi, 1983 fut déterminé comme une date charnière pour l'organisation puisqu'elle correspond au début du «programme clientèle» pour jeunes bénéficiaires de l'aide sociale. Il s'agissait, à partir de cette date, d'identifier l'impact des raccrocheurs en comparant la situation d'avant 1983 à celle qui existe maintenant. Cette comparaison eut lieu dans le cadre d'une seule étude de cas. Celle-ci fut effectuée en profondeur tout en la mettant en perspective avec des monographies d'école pour raccrocheur produite par le ministère de l'Éducation (Henripin et Proulx, 1986). Ce design de la recherche avait pour objectif de produire des connaissances pratiques et théoriques en regard de l'objet d'étude: identifier les concepts théoriques à l'œuvre tout en mettant en évidence le réseau intersubjectif, stratégique et symbolique dans lesquels ils s'actualisent.

Le cadre de référence qui a servi à la collecte des données s'est appuyé sur la notion de culture organisationnelle ${ }^{1}$, c'est-à-dire un ensemble de croyances et d'hypothèses partagées par les membres d'une organisation (Thévenet, 1986). La période de collecte des données s'est déroulée au cours de l'automne 1986. Les informations ont alors été recueillies par les méthodes suivantes:

- entrevues face à face avec des personnes clés;

- entrevues semi-structurées avec douze membres de l'organisation, aux fonctions diverses, ayant un vécu professionnel de la période avant 1983 et étant actuellement fortement impliqués auprès des publics raccrocheurs;

- collecte de données administratives, locales et provinciales, concernant les publics raccrocheurs;

- production de trois monographies de centres d'éducation des adultes de la Commission scolaire régionale Blainville-Deux-Montagnes impliqués auprès des raccrocheurs;

- observations terrains;

- revue de la littérature.

L'analyse et l'interprétation de ces informations ont fait l'objet d'une démarche interprétative telle que précisée plus haut. La signification des interactions entre l'organisme et les publics raccrocheurs étant au cœur de l'étude, un panel d'intervenants de premières lignes et d'autres davantage reliés aux diverses fonctions administratives fut constitué aux fins d'interagir avec le processus d'interprétation des données. Finalement, un rapport de recherche fut élaboré (Toupin, 1987). Celui-ci fut maintes fois réécrit et remodelé en tenant compte du profil d'intérêt des divers intervenants qui auraient à l'utiliser (ex.: commissaires d'écoles, gestionnaires, conseiller(ère) pédagogique, personnel enseignant).

\section{RÉSULTATS DE LA RECHERCHE}

Dans le processus d'interprétation des données, il est très vite apparu que l'impact des publics raccrocheurs était de deux ordres. D'une part, un impact de croissance qui, somme

1. Combinant les méthodes ethnographiques aux théories du développement organisationnel, la notion de culture organisationnelle permet de mettre en perspective l'histoire de l'organisme. ses signes et symboles, ses valeurs et surtout son métier, c'est-à-dire le sens que l'organisation donne à ses propres activités. La culture organisationnelle considère l'organisation sous l'angle de son identité ou style, plutôt que du modèle dont elle relève (ex.: public vs privé), de sa cohérence, plutôt que de ses normes, de son processus, plutôt que de son «produit». La culture organisationnelle apparaît ainsi comme un processus d'apprentissage continu où la tradition est constamment actualisée pour répondre aux problèmes et défis internes et externes. Par exemple, elle apparaitra de façon manifeste lors du processus de socialisation d'un nouvel enseignant face à certains problèmes reliés à l'enseignement, ou encore lorsqu'il s'agit de reconnaître un succès ou un échec et ainsi de suite. 
toute, ne dépend pas comme tel des caractéristiques des bénéficiaires de l'aide sociale âgés de 18 ans à 30 ans. D' autre part, un impact de spécificité qui, cette fois-ci, peut-être directement relié à la présence des jeunes raccrocheurs à l'éducation des adultes.

\subsection{IMPACT DE CROISSANCE}

Avec l'implantation du programme gouvernemental québécois d'insertion sociale et professionnelle des jeunes en 1983, les publics raccrocheurs n'ont cessé de croître aux Services de l'éducation des adultes de la Commission scolaire régionale Blainville-Deux-Montagnes. En 1982-1983, soixante places sont réservées pour les raccrocheurs dans deux centres, en 1983-1984, il s'agit de 80 places; en 1984-1985, avec l'apparition d'un nouveau modèle de financement du ministère de l'Éducation destiné à faire face à la pression de cette nouvelle clientèle, c'est 160 places réservées aux jeunes raccrocheurs que l'on dénombre, réparties dans trois centres. Finalement, en 1985-1986, deux places sur trois dans les centres d'éducation des adultes, soit environ 400 places, sont occupées par des jeunes raccrocheurs. Bien que les jeunes raccrocheurs ne représentent encore qu'une inscription sur cinq aux Services de l'éducation des adultes, c'est maintenant plus de $40 \%$ du total des heures de formation dispensées par les Services de l'éducation des adultes qui leur sont consacrées. Cette proportion est encore plus forte si l'on ne tient compte que des heures dispensées en formation générale.

La croissance des publics raccrocheurs a donc été rapide et concentrée. Seul les centres dispensant une formation générale ont connu cet impact. Bien que peu ou pas concernés par les jeunes raccrocheurs, les autres secteurs de l'éducation des adultes (ex.: formation professionnelle, formation agricole, éducation populaire) étaient eux aussi, durant la même période, légèrement en croissance. Dès lors, l'organisation centrale de l'éducation des adultes de type artisanal, ou "grande famille», où tous et toutes se connaissent ne pouvait plus exister. La croissance des services, due en bonne partie aux jeunes raccrocheurs, allait amener avec elle l'embauche de nouvelles personnes, l'expansion des locaux, une spécialisation accrue des tâches, une différenciation accrue dans les fonctions et l'apparition de nouvelles (ex.: coordination) ainsi qu'une segmentation du processus de la prise de décision jointe à l'apparition de prérogatives distinguant plus nettement gestionnaires et professionnels. Dorénavant, c'est la base administrative, notamment les activités de planification, de coordination et de contrôle qui marque le pas de l'organisation. Toutefois, parallèlement aux années 70 , les Services de l'éducation des adultes continuent à se définir comme «privatisés» ou «spécifiques», c'est-à-dire appliquant des règles et procédures propres à l'entreprise privée, constituant ainsi une enclave à l'intérieur d'une commission scolaire dont la majorité des effectifs sont consacrés à l'enseignement aux jeunes soumis à la fréquentation scolaire obligatoire. Ainsi, les programmes pour jeunes raccrocheurs étant octroyés par le gouvernement sous format d'annualitée ${ }^{2}$ l'organisation n'a pas cherché à profiter de cette croissance pour augmenter, dans les mêmes proportions, son bassin d'employés permarients. Les enseignants et enseignantes sont encore très majoritairement embauchés à la leçon à. l'éducation des adultes. De même, les services de marketing et de recrutement de la clientèle n'ont pas ralenti leurs activités puisque l'organisation est toujours en situation de concurrence par rapport à d'autres fournisseurs de services éducatifs (ex.: Cégeps, formation à distance, municipalités, firmes privées).

En résumé, l'augmentation importante de la fréquentation des jeunes raccrocheurs à l'éducation des adultes a suscité de profondes modifications, notamment au chapitre du financement et de la croissance des effectifs avec les ramifications organisationnelles que cela suppose. Il en est résulté un redéploiement accompagné d'une différenciation des fonctions administratives. Cependant, cette croissance organisationnelle a été intégrée au concept de «spécificité» de

2. Le programme pour jeunes raccrocheurs est sous la responsabilité du ministère de la Main-d'œuvre et de la Sécurité du revenu. Il est reconduit chaque année depuis maintenant quatre ans. Il se peut, et cela s'est déjà vu, que celui-ci soit aboli purement et simplement. L'éducation des adultes a déjà vécu de telles abolitions avec les conséquences que cela suppose: mise à pied, mise en disponibilité, réorganisation interne et ainsi de suite. Il n'est donc pas surprenant que les S.E.A. ne se risquent pas à créer des emplois permanents avec des programmes qui eux ne le sont pas. 
l'éducation des adultes, nonobstant le fait que les jeunes raccrocheurs sont encadrés par un programme d'origine gouvernementale.

\subsection{IMPACT DE SPÉCIFICITÉ}

Il ne faudrait pas croire que l'éducation des adultes ne s'est occupé des raccrocheurs qu'à partir de l'annonce des mesures gouvernementales en 1983. Les jeunes raccrocheurs fréquentent l'éducation des adultes depuis que ces services existent dans la plupart des commissions scolaires, c'est-à-dire depuis 1967. Ce qui est «nouveau», après la date charnière de 1983, c'est le caractère massif et concentré des publics raccrocheurs à l'éducation des adultes.

Auparavant, les jeunes raccrocheurs étaient disséminés ici et là parmi les publics «traditionnels» de l'éducation des adultes, par exemple les chômeurs et chômeuses, les femmes désirant effectuer un retour sur le marché du travail, les personnes en recyclage professionnel et les nombreux adultes dilettantes. L'éducation des adultes, ancienne manière, dépendait de deux grands courants qui ont fortement marqué les sociétés industrielles d'après guerre. D'une part, une demande soutenue de mise à jour professionnelle via un processus structurel et continu de déqualification-requalification. D'autre part, une demande importante de consommation culturelle reliée aux modifications dans les modes de vie (ex.: voyage tourisme, nouvelles philosophies du corps, changements dans les rapports familiaux ou familiers) et l'utilisation du temps personnel et social. Les adultes qui venaient à l'éducation des adultes selon l'un ou l'autre (ou les deux) de ces courants réclamaient une formation courte, ponctuelle et ad hoc. L'organisation devait offrir un produit qui visait juste en un laps de temps très court.

Depuis 1983, ces courants ont continué de s'affirmer mais ils n'occupent plus le devant de la scène. Plutôt, selon les termes mêmes des praticiens de l'éducation des adultes, c'est le phénomène du «rajeunissement des clientèles» qui retient dorénavant l'attention.

Les jeunes raccrocheurs ont des caractéristiques spécifiques qui sont connues depuis longtemps par les enseignants et enseignantes: manque d'autonomie, absence d'habiletés de base, faiblesse académique, problèmes sociaux-affectifs et autres. C'est souvent l'expression «échec scolaire» qui sert à recouvrir cette réalité multiple et plurielle. Auparavant, la problématique de l'échec scolaire était abordée de façon individuelle, quasi tutorale, à l'éducation des adultes. C'était des cas d'exceptions, vécus à la marge du système prévu pour la majorité des adultes. Les jeunes raccrocheurs devenant en l'espace de trois ans à peine, soit de 1983 à 1986, la «nouvelle majorité» en formation générale, les centres de formation ont dû repenser leurs orientations et organisations internes.

Aux dires des praticiens, avec les jeunes raccrocheurs, les plans de formation sont devenus plus longs. Les besoins de récupération académiques se sont accrus et il a fallu renforcer le suivi pédagogique. Chacune de ces mesures a eu un impact organisationnel certain. Ainsi, le phénomène important de la récupération académique a provoqué l'apparition d'un tout nouveau secteur à l'éducation des adultes: les classes dites de «pré-secondaire», classes qui sont à distinguer de celles qui sont destinées à l'alphabétisation. De même, l'importance du suivi pédagogique a provoqué le développement des fonctions périscolaires: conseiller(ère) d'orientation, conseiller(ère) aux étudiants(es), conseiller(ère) en information scolaire et professionnelle, orthopédagogue et autres. Finalement, la longueur des plans de formation a amené les centres de formation à modifier leur perception d'eux-mêmes. Dorénavant les jeunes qui fréquentent les centres de formation d'adultes veulent y retrouver une vie sociale interne pouvant susciter appartenance et identification. Les enseignants et enseignantes habitués qu'ils étaient à transiger avec des adultes relativement autonomes sont dorénavant confrontés à des phénomènes de groupes ou à des problèmes individuels (ex.: abus de drogue) pour lesquels ils ou elles se sentent peu préparés(es).

Ce sont là les impacts les plus observables de la présence désormais massive des jeunes raccrocheurs. Sur un plan plus qualitatif, les praticiens de l'éducation des adultes sont unanimes à dire que l'atmosphère des centres de formation n'est plus ce qu'elle était. Selon eux, la vie des centres est beaucoup plus structurée, voire même contrôlée. Cela tient d'une part aux mesures de contrôle qui accompagnent le programme pour jeunes raccrocheurs. Par exemple, même si le jeune raccrocheur a tendance à faire du centre un milieu de vie et d'appartenance, 
il est tenu, puisque le mode de financement le veut ainsi, de produire un certain rendement scolaire en fonction d'un échéancier précis. Bref, il n'est pas là pour se faire un autre chezsoi mais pour se réinsérer dans la société. D'autre part, outre ces mesures de contrôle imposées en quelque sorte de l'extérieur, les praticiens reconnaissent que l'éducation des adultes, du moins la formation générale, s'est elle-même durcie. «L'école est là pour scolariser» entendon dire de façon quasi unanime, tranchant ainsi radicalement avec l'idée de «déscolarisation» mise de l'avant au cours des années $70^{3}$. Face à une culture différente, celle des jeunes décrocheurs, les praticiens hésitent de moins en moins à affirmer la leur et à exiger que l'on s'y conforme. Le temps de la pédagogie adaptée semble révolu. Par exemple, les enseignants et enseignantes réaffirment avec force que leur rôle est d'enseigner et non pas de jouer au «psychologue», au «sociologue» et autres «logues».

La majeure partie du personnel enseignant ne craint plus d'assumer un rôle actif dans la «fabrication» de l'échec scolaire, puisque les règles du jeu sont les mêmes pour tous et toutes. Sur ce fond quasi unanime, certains enseignants et enseignantes sont prêts à faire un bout de chemin pour faciliter l'intégration des jeunes au curriculum scolaire. Si certains ne fonctionnent pas avec le régime pédagogique de l'éducation des adultes, il s'agirait environ du tiers des jeunes raccrocheurs selon une approximation qualitative, il faut, suggère-t-on, aménager des règles «allégées», menant à des sanctions académiques moindres.

Le personnel enseignant des Services de l'éducation des adultes de la Commission scolaire régionale Blainville-Deux-Montagnes n'est pas seul à tenir les propos. Les monographies du ministère de l'Éducation (Henripin, Proulx, 1986) sur les écoles pour jeunes raccrocheurs de mêrne que les études organisationnelles des commissions scolaires (Blackburn, 1986) révèlent des tendances similaires.

En résumé, l'impact spécifique des jeunes raccrocheurs s'est fait surtout sentir dans les centres de formation dispensant la formation générale. Le dispositif scolaire s'est transformé pour accueillir des jeunes moins autonomes et présentant un profil académique faible. Par contre, il semble que dans l'ensemble ce sont les jeunes raccrocheurs qui ont dû s'adapter au système d'enseignement plutôt que l'inverse. L'éducation des adultes s'est conceptuellement définie plus «scolarisante» que jamais. La scolarisation, concept refuge et sécurisant, met toutefois en perspective les profondes différences qui existent entre les raccrocheurs et les membres de l'organisation.

\section{IMPLICATIONS THÉORIQUES ET PRATIQUES}

Depuis le début en 1983, du programme pour les bénéficiaires de l'aide sociale âgés de 18 à 30 ans jusqu'au mois de juillet 1987, 46599 jeunes y avaient participé (source: ministère de la Main-d'œuvre et de la Sécurité du revenu, Direction des programmes). Des études évaluatives sont en cours visant à mesurer l'impact de ce programme de formation sur l'employabilité des jeunes décrocheurs. L'ambiguité sémantique des expressions «bénéficiaires de l'aide sociale» et «jeunes raccrocheurs» traduit bien les objectifs différents qui animent les dénominations institutionnelles, bien qu'il s'agisse en fait des mêmes personnes. Dans cette optique, il est nécessaire que l'éducation des adultes, en tant qu'organisme à responsabilité publique, tire ses propres évaluations de ce «programme clientèle» pour lequel elle est mandatée.

Les implications théoriques et pratiques du programme pour jeunes raccrocheurs sont nombreuses pour l'éducation des adultes de la Commission scolaire régionale Blainville-DeuxMontagnes. Certaines d'entre elles seront abordées ci-dessous. D'autres, à caractère plus

3. La Commission d'étude sur la formation des adultes (Commission Jean), dont le rapport final fut déposé en février 1982, avait cherché à traduire le principe de «déscolarisation» dans les pratiques de l'éducation des adultes tout en re rejetant pas les acquis de l'institutionnalisation. Faisant écho aux pratiques innovatrices présentes dans le champ de l'éducation des adultes des années 70, la Commission Jean préconisait, ni plus ni moins, de considérer l'adulte comme un être de pouvoir et d'autonomie. Conséquemment, les pratiques de formation devaient s'ouvrir, devenir flexibles et être décloisonnées pour accueillir les projets définis par les adultes. Cette pédagogie du projet, misant sur la prise en charge de l'adulte par lui-même, s'opposait à un «modèle scolaire» qualifié alors de normatif et mécaniste. 
local, ont été mises de côté. De plus, il faut aussi préciser que l'identification des implications dépend d'un processus qui est toujours en cours.

Sur le plan théorique, la problématique des jeunes adultes reste, et de loin, un terrain de recherche peu couvert. On ne peut certes pas parler de la socialisation de ces jeunes, de leur «intégration sociale» de la même façon qu'on en parle pour les enfants ou les adultes plus âgés. La reconnaissance du rôle nouveau joué par cette génération intermédiaire dans nos sociétés est pour le moment une problématique encore peu définie. De même, l'apparition de nouvelles formes d'analphabétisme chez ces jeunes issus de l'école de masse est un phénomène préoccupant. Peut-être plus préoccupant encore est la question de l'interaction jeune-adulte (Bruner, 1983). Tout semble se passer comme un double repli sur soi: repli des jeunes sur eux-mêmes et repli des adultes qui ne se voient pas ou peu comme accompagnateurs de ces jeunes, modèle d'engagement éthique et social, mais plutôt techniciens ou plombiers scolaires des «handicaps socioculturels». Comment, avec ces replis sur soi, une société peutelle réaliser les conditions de sa propre transformation? Une telle question rejoint minimalement le problème de la formation des maîtres et leur développement professionnel et, maximalement, la thématique plus large et englobante des nouveaux rapports individu-société à construire. Les «programmes clientèles» à la mode du jour laissent entrevoir que ces nouveaux rapports, dans le cas des jeunes raccrocheurs, sont plutôt instrumentaux. Ces rapports pourraient aussi être un processus de construction de l'autonomie de l'adulte.

Outre les interactions adultes-jeunes, d'autres implications théoriques doivent être mises en évidence. Ainsi, pour faire suite aux analyses macro-sociologiques des années 60 et 70 sur l'école comme lieu de sélection et de reproduction sociales, les années 80 et 90 ont besoin d'analyses plus fines, plus micro-sociologiques et davantage axées sur le processus que sur le résultat. C'est là l'intérêt entre autres de la «nouvelle sociologie» de l'éducation (Whitty, 1985).

Concernant le danger, réel ou appréhendé, d'une société duale, il devient impérieux d'examiner les rapports qu'entretiennent les jeunes adultes avec le savoir. Le savoir, avec l'influence des courants néo-conservateurs prônant «l'excellence», est à nouveau considéré comme neutre, objectif et universel. Si, sur un plan conceptuel, de telles caractéristiques sont possibles, il n'en demeure pas moins que les savoirs sont appropriés en fonction de considérations stratégiques, symboliques et affectives dont il est difficile de ne pas tenir compte dans une salle de classe. Les savoirs s'intègrent à une représentation du monde. Qu'elle est celle des jeunes adultes?

Cette même problématique des représentations pourrait aussi être appliquée à l'éducation des adultes comme tel. Il y a en effet un décalage certain entre la représentation mentale que se font les praticiens de ce qu'est maintenant l'éducation des adultes et ce qu'en disent les représentations publiques. Celles-ci en sont encore à présenter l'éducation des adultes à partir du discours «développementiste» qui a marqué les années 60 et 70 . L'heure n'est plus à la «participation», à la «démocratisation» ou à la «prise en charge». Plutôt, il s'agit maintenant de «socialiser», de «favoriser l'autonomie», de «scolariser». Il y a là une dynamique des représentations qui peut montrer à la fois le côté «contrôle social» des politiques en cours, mais aussi qu'il n'y a pas un rapport mécaniste entre les visées gouvernementales et ce qui est appliqué sur le terrain. Ainsi, l'éducation des adultes, malgré des marges de manœuvres externes réduites et une certaine adhésion interne à l'état d'esprit des années 80 , n'en continue pas moins à véhiculer certaines préoccupations et intentions à caractère humaniste ou constructiviste.

Les implications pratiques de l'impact des publics raccrocheurs sont de plusieurs ordres et niveaux. Sur un plan global, la montée des «programmes clientèles», notamment celui pour jeunes raccrocheurs, traduit une forte intégration de l'éducation des adultes aux politiques fiscales (ex.: sécurité du revenu) et de main-d'œuvre. Les objectifs de ces politiques, par exemple l'amélioration de l'employabilité, ont une saveur politique et sociale indéniable mais ils ne se traduisent pas facilement sur un plan éducatif. La formation, comme telle, ne conduit pas à l'employabilité. Elle peut aider un jeune adulte à sauter dans un train en marche mais ce n'est pas elle qui décide ou contrôle l'horaire des trains. Que signifie alors «améliorer l'employabilité» s'il n'y a pas une politique d'emploi sur laquelle vient se greffer les programmes 
de formation? De même, malgré un encadrement par les politiques fiscales et de main-d'œuvre, l'éducation des adultes peut-elle se développer de façon relativement autonome, en engageant sa propre responsabilité?

Sur le plan proprement éducatif, la présence massive des jeunes raccrocheurs pose bon nombre de problèmes au régime pédagogique de l'éducation des adultes. Il n'y a qu'un seul régime pédagogique de l'éducation des adultes, bien que celui-ci s'adapte au rythme de chaque adulte puisqu'il est individualisé. Les jeunes raccrocheurs ont de la difficulté avec l'enseignement individualisé. D'une part, ce type d'enseignement ne favorise pas la socialisation et, d'autre part, il faut être relativement autonome pour fonctionner en mode individualisé. Les jeunes raccrocheurs ayant souvent fréquenté des secteurs dévalorisés de l'enseignement régulier (ex.: professionnel court) se retrouvent dans un régime pédagogique à une seule voie pour lequel ils ou elles ne sont pas préparés(es). Il est fréquent de voir les jeunes raccrocheurs assumer de longues récupérations en français. Dès lors, une question pratique se pose: l'éducation des adultes doit-elle aménager des voies scolaires menant à différents types de sanctions et de diplômes? Doit-elle adopter une pédagogie plus «traditionnelle», par exemple magistrale, pour accommoder les jeunes raccrocheurs? Ces questions pratiques sont loin d'être faciles puisqu'elles impliquent un changement de cap important par rapport à ce que l'éducation des adultes croyait être son originalité et sa spécificité.

Un demier point pratique mérite aussi d'être mentionné. Jusqu'en 1983, l'éducation des adultes. n'avait jamais fait appel à l'appareillage de l'éducation spécialisée, par exemple aux orthopédagogues. Ce temps semble révolu. Les jeunes décrocheurs ont souvent de sérieuses difficultés d'apprentissage requérant une intervention spécialisée. Toutefois, et c'est là que le bat blesse, il n'y a peu ou pas de spécialistes connaissant les problèmes d'apprentissage des jeunes adultes. Un problème de dyslexie diagnostiqué à 5 ans ne se traite pas comme s'il était diagnostiqué à 20 ans. Le jeune adulte a développé plusieurs stratégies compensatoires dont il faut tenir compte et intégrer à l'intervention pédagogique.

Pour conclure, il est certain que les implications théoriques et pratiques tirées d'une seule étude de cas, même corroborées par des études organisationnelles parallèles ou semblables, ne peuvent être généralisées à l'ensemble des commissions scolaires du Québec. Bien que le phénomène des jeunes raccrocheurs soit présent dans toutes les commissions scolaires, il n'est pas sûr que toutes aient adopté les mêmes mesures et tiré les mêmes conclusions. Dês lors, il devient impérieux de définir des lieux pour confronter les interprétations et identifier les points de convergence et de divergence. Il serait peut-être alors possible de tirer des orientations susceptibles de favoriser le développement endogène de l'éducation des adultes dans le contexte d'une responsabilité sociale plus que jamais critique et vigilante.

\section{BIBLIOGRAPHIE}

BATIMION F. (1985), "In the Rush Toward Excellence don't Pet your Schools «Holding Power» Slide», American School Board Journal, 172 (10).

BLACKBURN F. (1986), Étude sur la clientèle du programme de rattrapage scolaire, Chicoutimi, C.S. Baie des $\mathrm{Ha}$ ! Ha!, Chicoutimi of Valin, Service de l'éducation des adultes.

BRODEUR D. (1977), Étude de la situation de chomage des jeunes et de la situation d'intervention au Québec, Montréal, École nationale d'administration publique.

BRUNER J. S. (1983), Savoir faire, savoir dire, Paris, PUF.

CAMPEAU J. C. (1985), Études d'évaluation sur les mesures de relance et la participation des jeunes bénéficiaires de l'aide sociale, Montréal, ministère de la Main-d'œuvre et de la Sécurité du revenu.

CUNGEMI J. P. et D. L. COAN (1973), «A Clinical Method for Identifying Potential Dropouts», College Student Journal, 7 (1).

DEMARTINI J. R. et L. B. WHITBECK (1986), «Knowledge Use as Knowledge Creation: Reexaming the Contribution of the Social Sciences to Decision Making», Knowledge, 7 (4).

DEMERS M. (1984), «Les conséquences psychologiques et sociales du chômage chez les jeunes: revue de la littérature», dans M. C. Lamarche (édit.), Actes du colloque sur la recherche sociale 1983, Québec, ministère des Affaires sociales, Conseil québécois de la recherche sociale. 
DUBOIS E. (1985), la Situation des jeunes de l'aide sociale, Montréal, ministère de la Main-d'œuvre et de la Sécurité du revenu.

FORREST B. (1986), «The Store Front School», Guidance and Counselling, 1 (4).

GOUVERNEMENT DU QUÉBEC (1984), Livre blanc sur la fiscalité des particuliers, Québec, Éd. officiel.

HÉBERT J. et P. YUZYK (1986), Jeunesse: un plan d'action, Ottawa; gouvernement du Canada.

HEDMAN, C. et M. HORNING (1983), «Success with High School Dropouts», Educational Leadership, 40 (6).

HENRIPIN M. et L. PROULX (1986), les Écoles de chances: l'école le Virage, Québec, ministère de l'Éducation.

ID. (1986), les Écoles de chances: l'école Boudreau, Québec, ministère de l'Éducation.

ID. (1986), les Écoles de chances: La nouvelle école, Québec, ministère de l'Éducation.

JONES L. H. et H. MAROCKIE (1987), «Reducing Dropout Rates through Home-School Communication», Education and Urban Society, 19 (2).

JUSTIZ M. et M. KAMEEN (1987), «Business Offers a Hand to Education», Phi Delta Kappan, 68 (5).

LEMONDE, M. (1986), le Concept de l'employabilité et les jeunes, Montréal, ministère de la Main-d'cuvre et de la Sécurité du revenu.

POLANYI M. (1958), Personal Knowledge, Chicago, University of Chicago Press.

SCHIMDT V. (1987), «Four Approaches to Science and their Implications for Organisational Theory and Research», Knowledge, 9 (1).

SERVOIN F. et R. DUCHEMIN (1986), Inégalités et solidarités, Paris, ESF.

THEVENET M. (1986), Audit social de la culture d'entreprise, Paris, Éd. de l'Organisation.

TOUPIN L. (1987), Impact des publics I.S.P.J. sur la culture organisationnelle des services de l'éducation des adultes de la Commission scolaire régionale Blainville-Deux-Montagnes, Saint-Eustache, Commission scolaire régionale Blainville-Deux-Montagnes, S.E.A.

WITTY G. (1985), Sociology and School Knowledge, Londres, Methuen.

YIN R. K., P. G. BATEMAN et G. B. MOORE (1985), «Case Studies and Organizational Innovation: Strengthening the Connection», Knowledge, 6 (3).

YIN R. K. (1981), "The Case Study as a Serious Research Strategy», Knowledge, 3 (1).

ID. (1984), Case Study Research: Design and Methods, Beverly Hills, Sage.

\section{RÉSUMÉ}

Après trois ans d'existence, les Services de l'éducation des adultes de la Commission scolaire régionale BlainvilleDeux-Montagnes examinent l'impact que le programme de formation pour jeune bénéficiaire de l'aide sociale a eu sur l'organisation scolaire. On a réalisé une étude de cas, qualitative et exploratoire. Elle permet d'interpréter et d'appréhender de façon contextuelle un certain nombre de problèmes actuels tel celui du rapport entre jeunes et adultes et le recours de plus en plus fréquent aux programmes centrés sur une clientèle dans l'organisation scolaire.

\section{SUMMARY}

After its first three years of existence, the adult education services of the regional school board of Blainville-DeuxMontagnes examine the impact that its training program for young welfare recipients has had on school organization. A case study, qualitative and exploratory in nature was realized, which makes it possible to interpret and apprehend, in a contextual way, a certain number of current problems such as youth-adult relationships and the more and more frequent recourse to "clientele centered programs" in school organization.

\section{RESUMEN}

Después de tres años de existencia, los Servicios de educación para adultos de la Comisión escolar regional BlainvilleDeux-Montagnes examinan el impacto que ha tenido el programa de formación para los jóvenes beneficiarios de la ayuda social sobre la organización escolar. Resultó un estudio de casos, cualitativo y exploratorio, que permite interpretar y captar de manera contextual un cierto número de problemas actuales tal como aquel de la relación jovenadulto y el recurso cada vez más frecuente a los "programas centrados sobre las clientelas" en la organización escolar. 